\title{
Dynamic Mri of the Cervical Spine - An Important Tool in Planning Surgical Treatment of Cervical Compressive Myelopathy
}

\author{
Sibhi Ganapathy ", Venkataramakrishna Tukapuram, Nikunj Godhani, Swaroop Gopal \\ Department of Neurosurgery, Sakra Institute of Neurosciences, Bangalore, India
}

Email address:

sibhig@yahoo.co.uk (S. Ganapathy)

${ }^{*}$ Corresponding author

\section{To cite this article:}

Sibhi Ganapathy, Venkataramakrishna Tukapuram, Nikunj Godhani, Swaroop Gopal. Dynamic Mri of the Cervical Spine - An Important Tool in Planning Surgical Treatment of Cervical Compressive Myelopathy (CONFIRMED). International Journal of Neurosurgery.

Vol. 2, No. 1, 2018, pp. 17-22. doi: 10.11648/j.ijn.20180201.14

Received: June 6, 2018; Accepted: July 2, 2018; Published: July 26, 2018

\begin{abstract}
Cervical compressive myelopathy (CM) remains a common entity in the practice of spine surgery. Though MRI is the diagnostic modality for CM, there are a subset of patients whose clinical features are suggestive of multilevel compression caused by dynamic factors, which however cannot be detected by a static MRI study. The causes include buckling of ligamentum flavum, collapse of disc height and changes in posterior longitudinal ligaments at the level of compression. These changes can only be detected on dynamic MRI. The application of Dynamic MRI can reveal not just additional levels of compression but even the site of maximum pressure, thus playing a vital role in surgical planning. It is also seen that adjaent level disease, commonly thought to be secondary to Cervical fusion was actually pre-existing and could have been picked up if dynamic MRI were used. We thus planned a prospective cohort of 24 patients of multilevel cervical compessive myelopathy who were studied with dynamic MRI at the Sakra Spine centre in Bangalore India. The clinical symptoms were correlated to the dynamic changes in MRI and were considered for planning appropriate treatment. Of the 24 patients in our study, 17 patients had a change in the original plan of treatment, either between conservative therapy and surgery, or the approach (Anterior vs. Posterior) of surgery. It was also noted that additional levels of compression discovered on the dynamic MRI were instrumental in changing the treatment protocol. Thus Flexion and Extension MRI is an important tool in planning the appropriate management in cervical compressive myelopathy.
\end{abstract}

Keywords: Cervical Compressive Myelopathy, MRI, Ligamentum Flavum, Surgery

\section{Introduction}

Cervical compressive myelopathy (CSM) remains a common entity in the practice of the spine surgery [1]. Though MRI is the diagnostic modality of choise for CSM, there is one sub group of patients whose cervical compressive myelopathy are due to multilevel compression caused by dynamic factors such as buckling of ligamentum flavum, collapse of disc height, or changes in posterior longitudinal ligaments at the level of compression. This can be picked up only on dynamic MRI [1].

Few studies have been done to understand this concept. But all the studies showed that the diameter of the canal is reduced in extension $[1,2]$. So far, no studies have been done to correlate these findings to assist the decision making process in cervical spondylotic myelopathy.

Another bleak fact is the increased emergence of adjacent level disease in fusion patients, both in the cervical and lumbar regions. The Severe extent of compression and sometimes instability despite adequate fusion and decompression coupled with accelerated rates of ligamentum flavum hypertrophy and osteophyte synthesis beggars belief. It has been postulated, that the origins of this disease lie in the earlier MRI prior to the first surgery, a fact that is well demonstrated on dynamic MRI.

Thus we attempted to document the effects of Dynamic MRI in management of cervical myelopathy, emphasizing on the changes in treatment protocol effected by the new findings discovered. 


\section{Materials and Methods}

All patients presenting with cervical compressive myelopathy were studied over a period of 1 year at the Spine Centre, Sakra Institute of Neurosciences, Bangalore. A total of 24 candidates were included in this study as a prospective observational cohort. All Patients were informed about the nature of the study and the extra imaging which would be performed before deciding upon therapy. A full informed consent was taken before subjecting them to a dynamic MRI. The MRI was done to the extent permittent by the spines of the patient without causing dificulty or deficiets. The change in treatment protocol after the dynamic MRI was informed to the patients before subjecting them to surgery and physiotherapy.
The patients ranged from 25 years to $79 y$ yrs of age of which 22 were male. Compression in the cervical spine was analysed with Dynamic MRI and the implications to patient management were documented. The MRIs were taken in flexion and extension in all patients without worsenning their symptoms, before analysing the clinical presentation, radiology and other factors such as age, and co-morbidities.

\section{Results}

\subsection{Demographics}

Most of the patients with CM were under the age group of 55 - 75 years. Male to female ratio was 11:1.

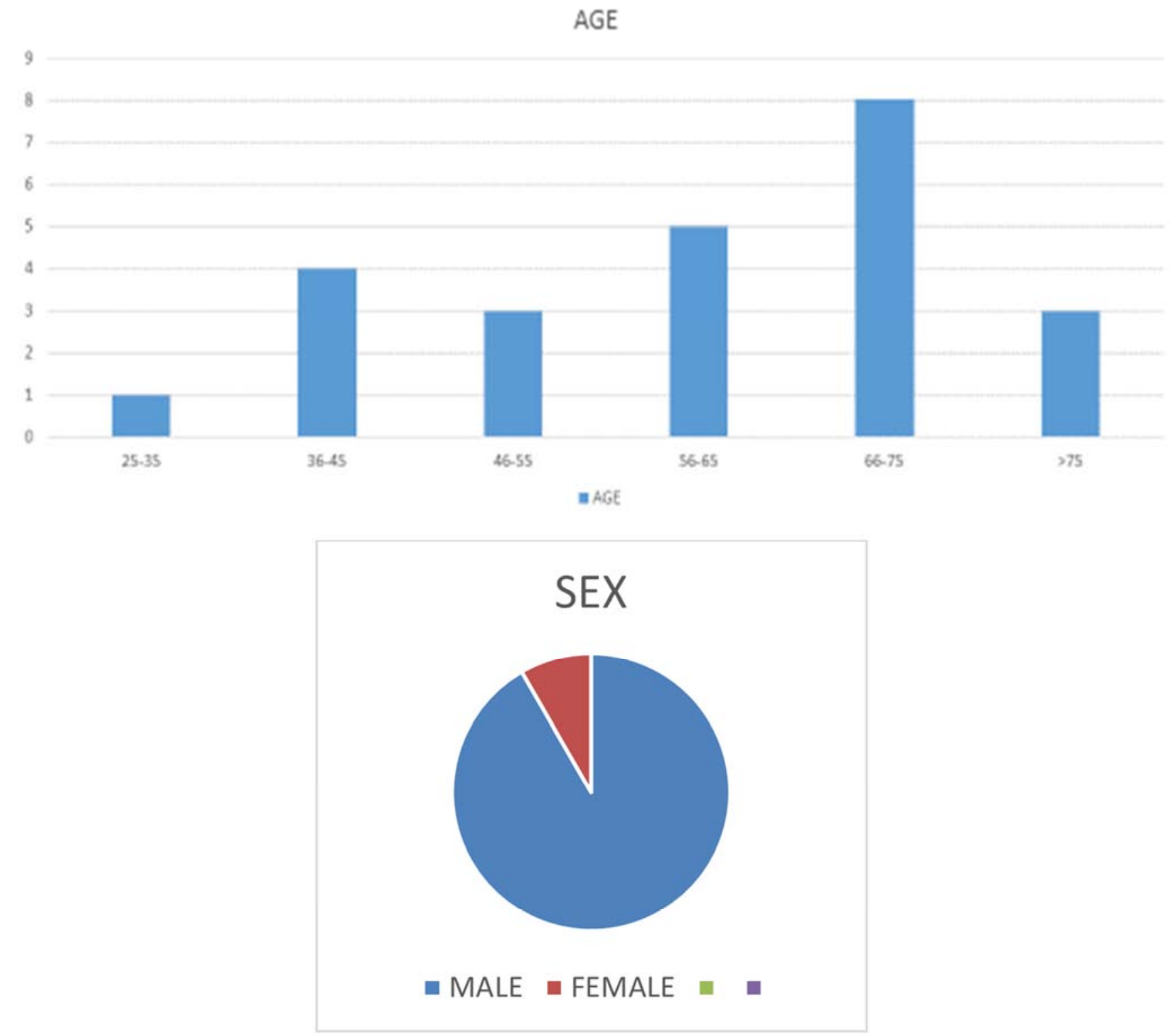

Figure 1. Age distribution and gender profile.

Total number of levels of compression were 59 and the additional levels of involvement were 37 . Additional levels of compression was noted in 17 patients and the average no. of additional levels were 1.54 per patient. All additional levels of compression were noted in extension. 


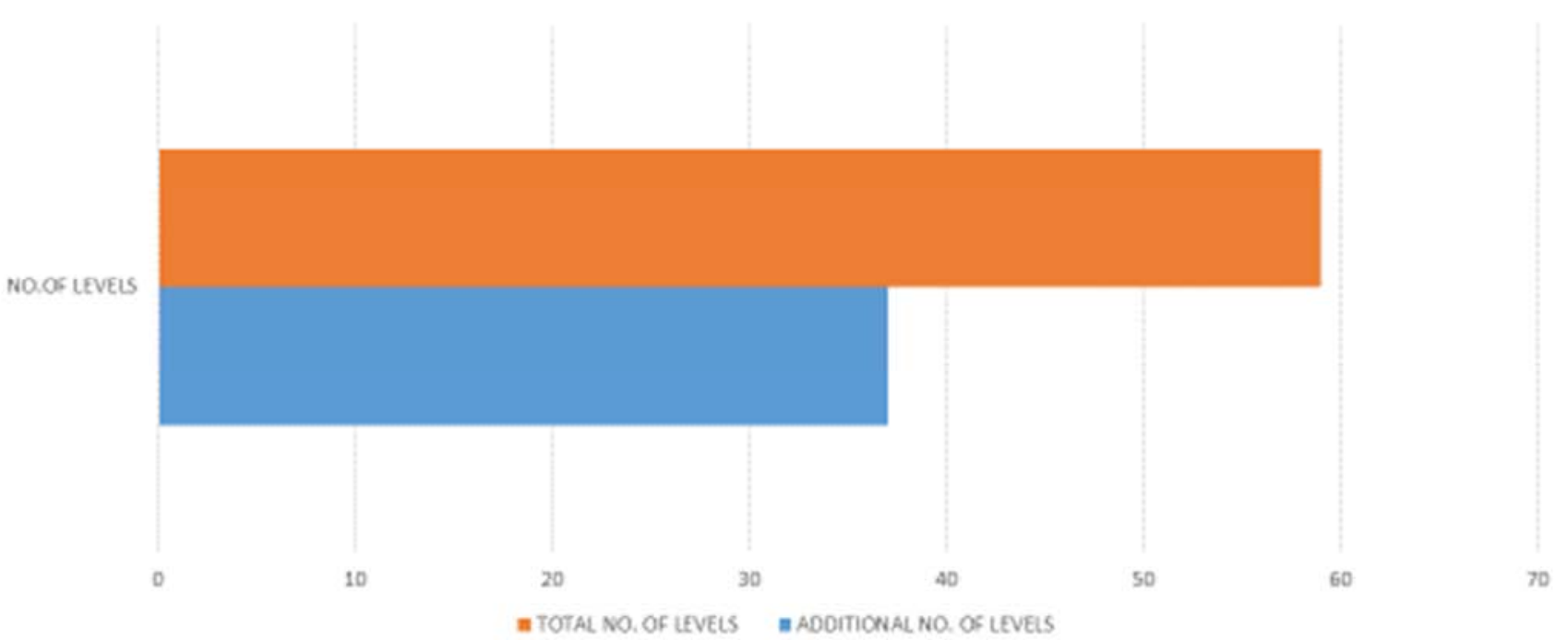

Figure 2. Number of involved levels and additional levels detection dynamic mri.

Surgery was considered in 20 patients. Posterior surgeries were 11 (laminoplasty/ laminectomy+lateral mass fusion), and anterior procedures (ACDF/ corpectomy and fusion) were 9. The rest of the patients did not require surgery and was conservatively treated. However, all the conservatively treated cases were regularly follwed up to look for any progression of the symptoms or deterioration.

\subsection{Change in Strategy: Management protocols Were Changed in 16 Patients}

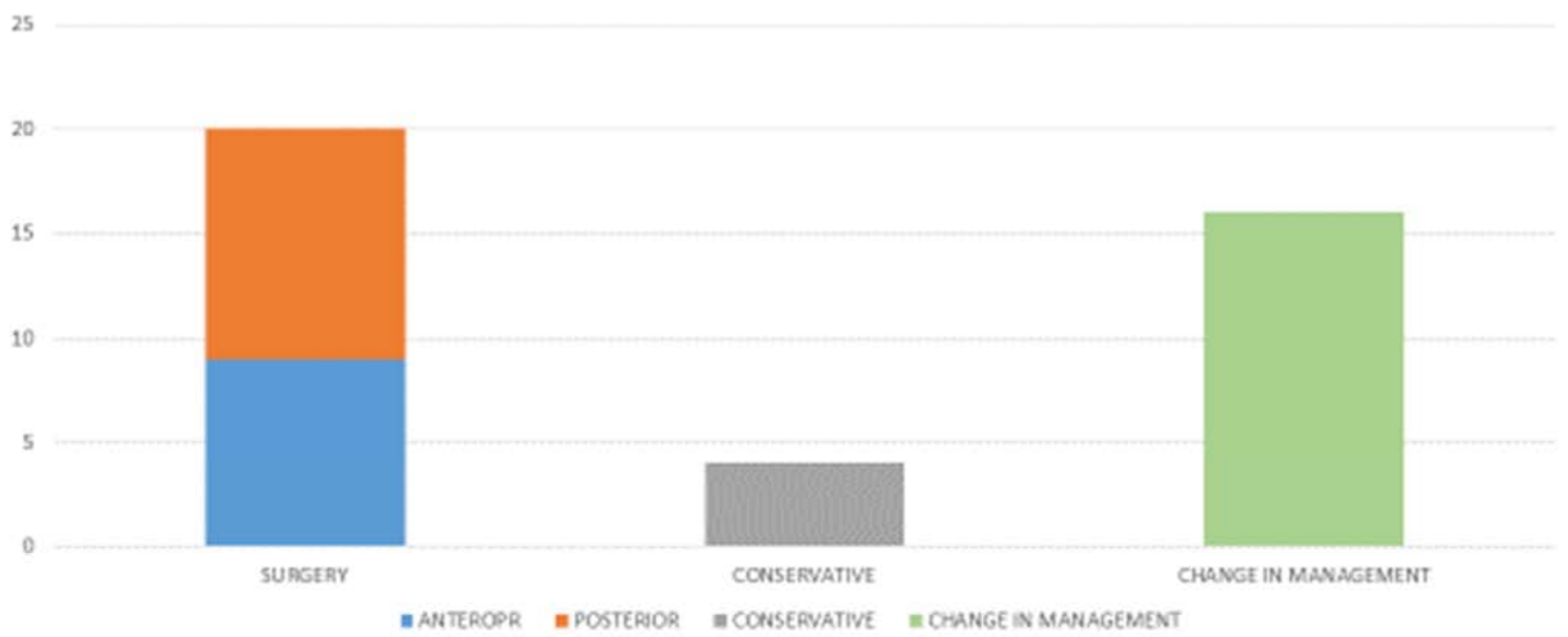

Figure 3. Profile of management strategies employed.

\subsection{Case Examples}

\subsubsection{Case 1}

A 35 yrs old male patient presented with b/1 upper limb numbness and difficulty in walking. On physical examination, the patient had spastic gait and a positive hoffmann's test bilaterally. A static MRI showed myelomalacia at C6-7 level.

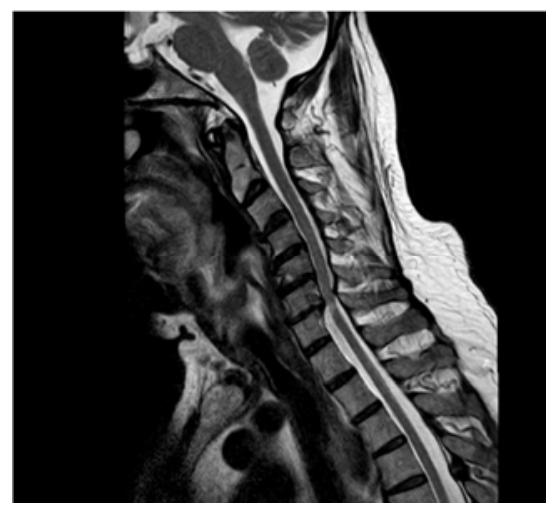




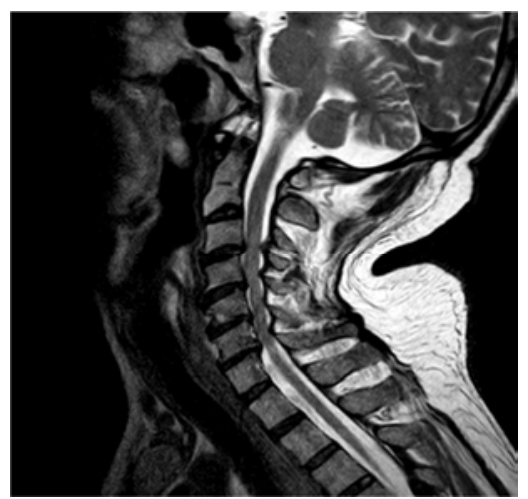

Figure 4 Dynamic MRI Cspine of case 1.

As demonstrated in the Figure 4, the extension showed a significant increase in the number of levels involved resulting in a change in procedure employed.

TREATMENT: C3 - C6 LAMINECTOMY + LATERAL MASS FIXATION. (AS THERE IS LOSS OF CERVICAL LORDOSIS), as opposed to a single level procedure.

\subsubsection{Case 2}

A $50 \mathrm{yrs}$ old female patient presented with features of CSM MRI showed myelomalacia at C3-4 level.

As seen in figure 5 there are 3 additional levels of compression on the cord, which is due to buckling of ligamentum flavum and changes in PLL.
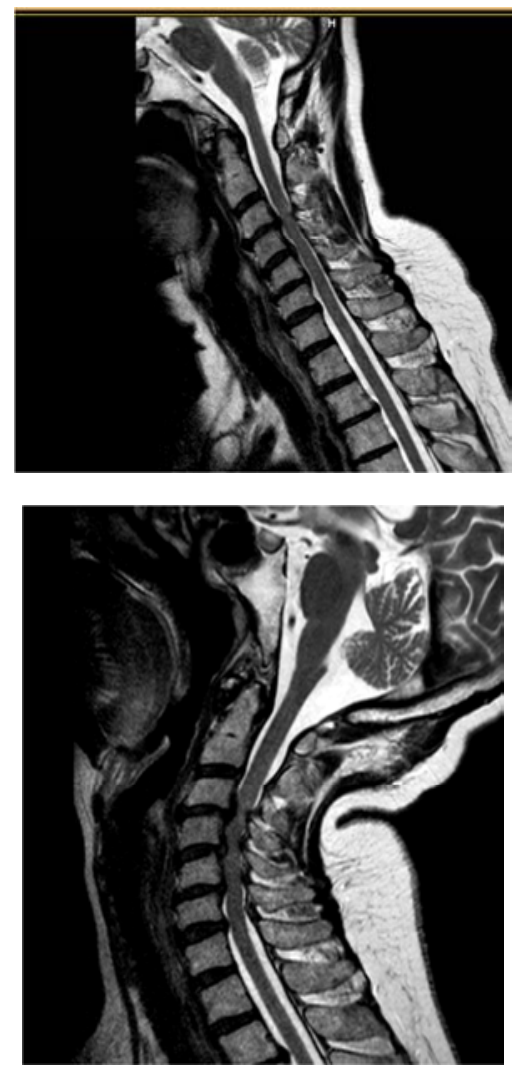

Figure 5 Dynamic Cervical MRI of case 2.

TREATMENT: C3 - C6 LAMINOPLASTY as opposed to a single level $\mathrm{ACDF}$ at $\mathrm{C} 34$

\subsubsection{Case 3}

A 55 yrs old male patient presented with features of CSM with significant posterior column involvement.

MRI showed myelomalacia at C4-5 level.

The Dynamic MRI as shown in figure 6 revealed additional 1 level of compression on the cord, which is due to buckling of ligamentum flavum and changes in PLL.
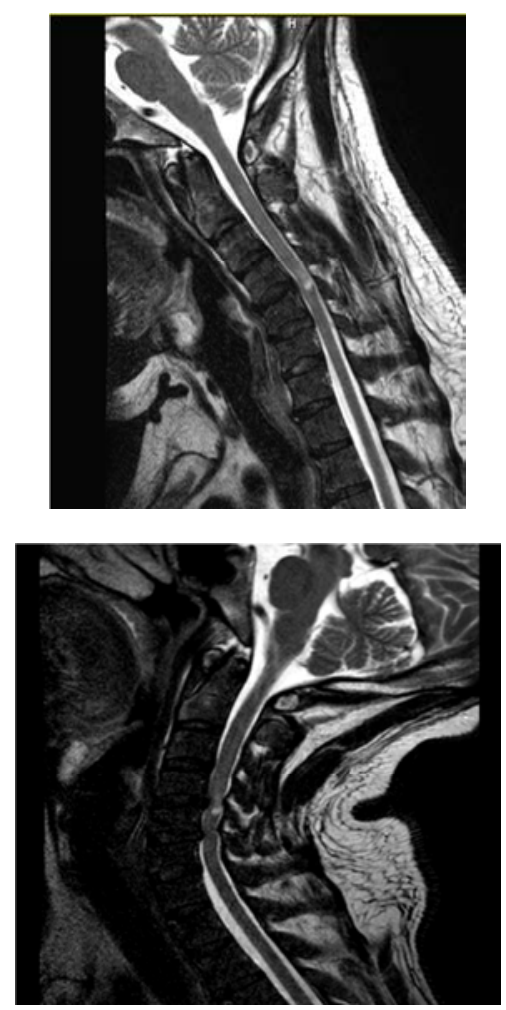

Figure 6. Dynamic Cervical MRI of case 3.

TREATMENT: C4-5 and C5-6 ANTERIOR CERVICAL DISCECTOMY AND FUSION as opposed to a single level ACDF originally considered.

\subsubsection{Case 4}

A 55 yrs old male patient presented with features of CSM MRI showed myelomalacia at C5-6 level.

Extension MRI revealed additional 1 level of compression on the cord, which is due to buckling of ligamentum flavum and changes in PLL. This is shown in Figure 7

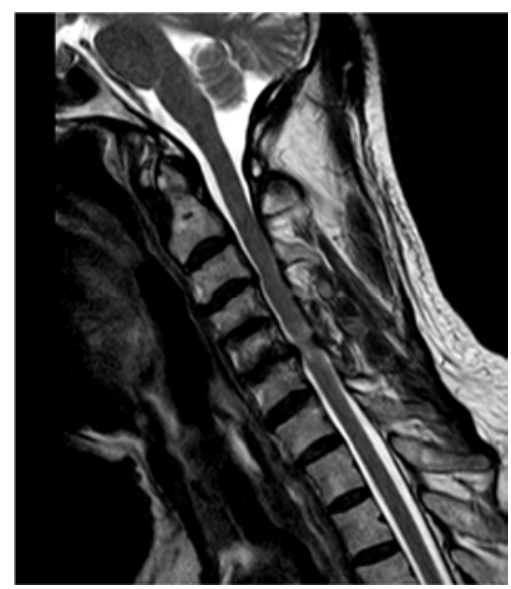




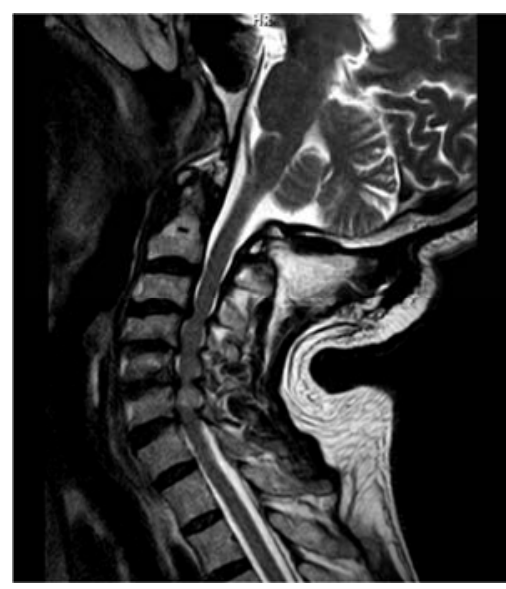

Figure 7. Dynamic Cervical MRI of case 4.

TREATMENT: C3 - C6 LAMINOPLASTY as opposed to a single level procedure.

\subsubsection{CASE 5}

A 56 yrs old male patient presented with weakness in the upper limbs with wasting of thenar muscles and interossei weakness. There were no signs of myelopathy.

Dynamic MRI demonstrated increasing severity of compression on extension with anterior compression due to disc osteophyte complex and posteriorly due to buckling of ligamentum flavum as seen in figure 8
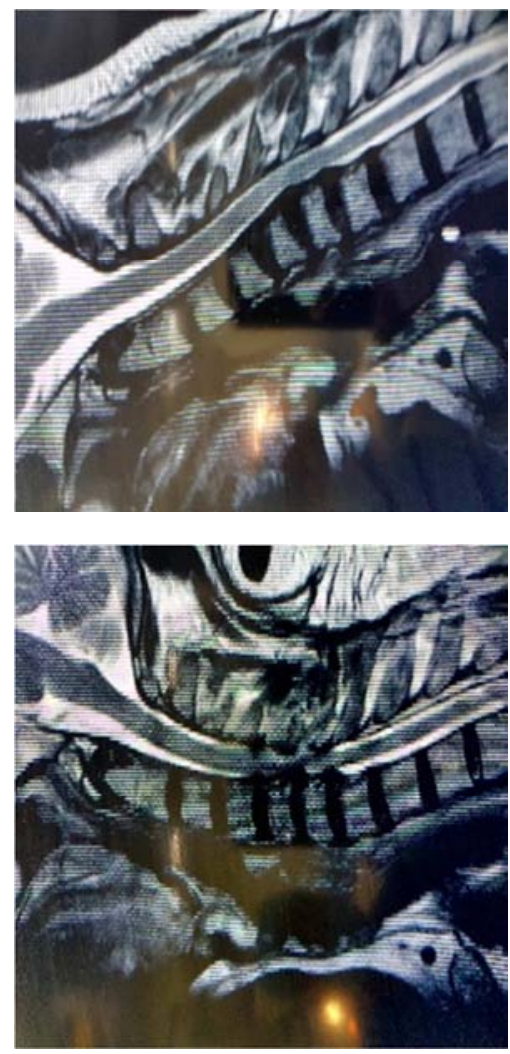

Figure 8. Dynamic Cervical MRI of case 5.

TREATMENT: C5, C6 CORPECTOMY AND C4-C7 STABILISATION, accounting for the anterior as well as posterior compression and curvature of the spine.

\section{Discussion}

Although MRI is a useful tool for diagnosis of CM, it does not give an exact idea as to which is the offending level in a multilevel compression that requires surgery $[1,2]$. Even the approach and procedure cannot be decided on a static examination and hence are subject to significant interpractitioner variability. Factors causing the CSM are also thought to be dynamic which change with the position of the spine and thereby impact the cord in a mobile manner. [2-5].

There are studies where the difference in the canal dimensions in cervical spine, are demonstrated unambiguously. [2, 3] Change in spinal cord cross sectional area, caused by ligamentum flavum buckling and vertebral disc protrusion have been seen associated with decrease in the canal anterio - posterior diameter on extension [3, 4].

Pathological changes in the cord were studied on MRI by Morshita and associates and formed the basis of a classification of cervical myelopathy patients based on the spinal canal diameter size, and, a relation between disc degeneration and cord compression.[4] Edward et. al used plain $\mathrm{X}$ rays for the measurements, which were taken from the posterior surface of the vertebral body to the spinolaminar line [4]

To avoid magnification variables, Pavlo et al. used the ratio of sagittal diameter of spinal canal to the sagittal diameter of vertebral body as a determinant of spinal canal stenosis. $[4,6]$

However, MRI has an advantage of showing soft tissue along with the bony components, dural compression, myelomalacia changes, Cervical cord compression, obliteration of subarachnoid space due to disc herniation, ostephyte formation as well as hypertrophy of the ligamentum flavum. [4-11]

None of the previous studies showed a correlation between symptomatology and the radiological compression detected by the Dyanmic MRI. [3-10] We have tried to show how the dynamic MRI changes the surgical planning in patients of CSM. The dynamic factors for the compression noted included, buckling of ligamentum flavum, collapse of disc height, and changes in posterior longitudinal ligaments at the level of compression. [6-11]

24 patients were enrolled in our study had degenerative compressive myelopathy. They had single to multiple level compression. The factors responsible for the compression were apparent in extension of the neck. On subjecting these patients to dynamic MRI study, they showed additional 1.54 levels of significant compression per patient on an average in extension views. Most of the symptomatic patients were under the age group of $50-65$ yrs. Which is consistent with other data concerning the incidence of cervical myelopathy. The number of additional levels of compression were 37 which were noted in a total 17 patients. Surgery was done in 20 cases of which, of which anterior procedures were considered in 9 cases. Here even a single level ACDF will not only remove anterior 
compression but also posterior compression by preventing buckling of ligamentum flavum due to increasing the disc height by using grafts. Corpectomy was done in cases where more than one level of anterior compression was present, especially in young patients. Posterior procedures were done in 11 patients where in MRI, the extension showed multilevel involvement with a predominant posterior component. In the elderly, all compression was treated by posterior procedures.

\section{Conclusion}

Flexion and extension MRI is an important tool for decision making and planning appropriate management in cervical compressive myelopathy. We recommend it to should be an investigation of choice in all patients of CSM.

What changed when we used Dynamic MRI? The extent of the whole disease was apparent: For the first time, hidden levels of compression made apparent on dynamic MRI could be treated appropriately. These would have been missed in the regular Static MRI. The missed levels of compression would over a period of 6 months to 2 years present as the dreaded adjacent level disease, which was thought to be a response to the redistribution of stress post fusion. Hence by using the Dynamic MRI, pre-existing hidden compression could be addressed satisfactorily.

With the complete extent and site of compression finally visible, the approach to surgery became apparent. Various changes in approach, both surgical and medical, allowed better comprehensive management of the disease ensuring better outcomes and happier patients.

\section{Conflict of Interest}

The authors declare that no conflict of interest exists

\section{Acknowledgements}

We would like to thank Dr. Srikant Narayanaswamy MD, Consultant Radiologist, Sakra Department of Radiology for his assistance and insight.

\section{References}

[1] Zhang L, Zeitoun D, Rangel A, Lazennec JY, Catonné Y, Pascal-Moussellard H. Preoperative evaluation of the cervical spondylotic myelopathy with flexion-extension magnetic resonance imaging: about a prospective study of fifty patients. Spine. 2011 Aug 1; 36 (17):E1134-9.
[2] Machino M, Yukawa $\mathrm{Y}$, Ito $\mathrm{K}$, Nakashima $\mathrm{H}$, Kato $\mathrm{F}$. Dynamic changes in dural sac and spinal cord cross-sectional area in patients with cervical spondylotic myelopathy: cervical spine. Spine. 2011 Mar 1; 36 (5):399-403.

[3] Harada T, Tsuji Y, Mikami Y, Hatta Y, Sakamoto A, Ikeda T, Tamai K, Hase H, Kubo T. The clinical usefulness of preoperative dynamic MRI to select decompression levels for cervical spondylotic myelopathy. Magnetic resonance

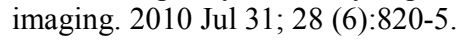

[4] Morishita Y, Naito M, Hymanson H, Miyazaki M, Wu G, Wang JC. The relationship between the cervical spinal canal diameter and the pathological changes in the cervical spine. European Spine Journal. 2009 Jun 1; 18 (6): 877-83.

[5] Lebl DR, Hughes A, Cammisa FP, O'Leary PF. Cervical spondylotic myelopathy: pathophysiology, clinical presentation, and treatment. HSS journal. 2011 Jul 1; 7 (2):170-8.

[6] Kuwazawa Y, Bashir W, Pope MH, Takahashi K, Smith FW. Biomechanical aspects of the cervical cord: effects of postural changes in healthy volunteers using positional magnetic resonance imaging. Clinical Spine Surgery. 2006 Jul 1; 19 (5):348-52.

[7] Henderson FC, Geddes JF, Vaccaro AR, Woodard E, Berry KJ, Benzel EC. Stretch-associated injury in cervical spondylotic myelopathy: new concept and review. Neurosurgery. 2005 May 1; 56 (5):1101-13.

[8] Bakhsheshian J, Mehta VA, Liu JC. Current Diagnosis and Management of Cervical Spondylotic Myelopathy. Global Spine J. 2017 Sep; 7(6): 572-586. Published online 2017 May 31.

[9] Lu K, Gao X, Tong T, Miao D, Ding W, Shen T. Clinical Predictors of Surgical Outcomes and Imaging Features in Single Segmental Cervical Spondylotic Myelopathy with Lower Cervical Instability. Med Sci Monit. 2017; 23: 36973705. Published online 2017 Jul 30

[10] Zhang C, Das SK, Yang DJ, Yang H. Application of magnetic resonance imaging in cervicalspondylotic myelopathy. World J Radiol. 2014 Oct 28; 6(10): 826-832. Published online 2014 Oct 28.

[11] Ellingson BM, Salamon N, Holly NT. Advances in MR Imaging for Cervical Spondylotic Myelopathy. Eur Spine J. 2015 Apr; 24 (Suppl 2): 197-208. Published online 2013 Aug 6. 